\title{
La motivación y el estudio de la función cuadrática con GeoGebra@
}

\section{The motivation and the study of the quadratic function with GeoGebra $₫$}

\begin{abstract}
Ramiro Rodríguez-Mendoza (D)
Universidad Distrital Francisco José de Caldas, Bogotá, Colombia

Oscar Jardey Suárez

Universidad Distrital Francisco José de Caldas, Bogotá, Colombia

\section{Resumen}

Objetivo: Describir las variables subyacentes de la motivación y estilos atribucionales de los estudiantes de educación básica de un colegio privado en Bogotá-Colombia, cuando estudian la función cuadrática apoyados en el software de Geometría dinámica GeoGebra®. Método: Con enfoque metodológico mixto. Se utiliza el inventario CEAP-48, de forma adicional se realiza una entrevista a un grupo focal. Los resultados se analizan bajo el Análisis de componentes principales con la confiabilidad interna de coeficiente global a de Cronbach. Resultados: El examen como motivación y el aprendizaje y su utilidad se mantienen como variables subyacentes a la motivación, en tanto que la calificación como motivación emerge como variable después de la implementación de la Secuencia Didáctica. GeoGebra $\Re$, en el decir de los estudiantes, es un elemento de motivación y mediación toda vez que permite experimentar, comprobar y visualizar algunas conjeturas. Discusión y Conclusiones: Las categorías propias del instrumento CEAP-48 muestran los mismos resultados en el pretest y postest de la investigación como en el de los autores del mismo, lo que indica que el instrumento tiene una validez diacrónica en el tiempo. Además, el examen hace parte de la motivación del estudiante, logrando afectar sus emociones en la academia.
\end{abstract}

Palabras clave: Educación matemática, e-learning, enseñanza a distancia, función cuadrática, GeoGebra®.

\begin{abstract}
Objective: Describe the underlying variables of motivation and attributional styles of elementary school students in a private school in Bogotá-Colombia, when they study quadratic function supported by GeoGebra ${ }^{\circledR}$ dynamic geometry software. Method: The methodological approach is a mixed one. The CEAP-48 inventory is used, in addition a focus group interview is conducted. The results are analyzed under the Main Component Analysis with the internal reliability of Cronbach's global coefficient a. Results: The test as motivation and learning and its usefulness remain as variables underlying motivation, while the score as motivation emerges as a

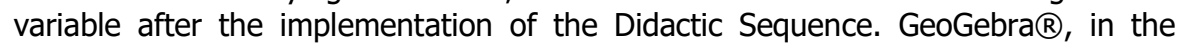
students' words, is an element of motivation and mediation since it allows to experiment, check and visualize some conjectures. Discussion y Conclusion: The categories of the CEAP-48 instrument show the same results in the pre-test and posttest of the research as in the authors', which indicates that the instrument has a diachronic validity in time. Moreover, the test is part of the student's motivation, managed to affect their emotions in the academy.
\end{abstract}

Keywords: Mathematical Education, e-learning, distance learning, quadratic

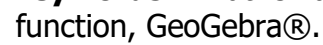

Open Access:

ISSN: $0124-2121$ E-ISSN: $2665-2420$

Editor: Dhayana Fernández Matos 


\section{Introducción}

La motivación es un factor al que la escuela tradicional no ha logrado dar total respuesta, por tanto, se hace necesario que el niño explore nuevas estrategias como aprender en escenarios lúdicos (Núñez \& González, 2020) e inmerso en la tecnología, la cual se constituye como una realidad innegable en la sociedad actual. Es así como la escuela ha venido transformando su manera de actuar en la enseñanza-aprendizaje, relacionando además, los medios digitales (Gómez, Rodriguez \& Marín, 2020). En tanto, el avance tecnológico ha permeado el quehacer docente, exigiendo estar a la vanguardia de las nuevas formas de concebir el mundo y las realidades de los niños y de esta manera aprovecharlo y utilizarlo a su favor en el aula de clases.

En un sentido estricto y etimológico, la motivación se refiere a movimiento, que tiene virtud para mover, envuelve una necesidad que puede ser originada desde lo psicológico o lo fisiológico (Carrillo, Padilla, Rosero \& Villagómez, 2009). Existen dos tipos de motivación; interna y externa; Elizondo, Rodríguez \& Rodríguez (2018) muestran que la primera está relacionada con estímulos internos que buscan satisfacer deseos del sujeto y es por tanto que existe una búsqueda autónoma del aprendizaje, para poder entender algo que antes no tenía, mientras que la segunda, está vinculada con factores externos como recompensas o castigos (Zull, 2002), que se traduce como motivación intrínseca y motivación extrínseca.

Para identificar los cambios motivacionales, se utiliza el test CEAP-48 propuesto por Barca, Porto, Santorum, Brenlla, Morán \& Barca (2005) en el que existen dos escalas: SEMAP-01, que muestra la motivación académica y SEAT-01, que estudia las variables atribucionales en relación al éxito académico. Las categorías propias del instrumento permiten entender la motivación académica desde lo extrínseco, intrínseco y superficial, recogidas en algunos de sus ítems y haciendo uso del Alfa de Cronbach para su consistencia. Resulta relevante reconocer y contrastar el comportamiento de los Alfa antes (pretest) y después (postest) de la intervención pedagógica que relaciona la tecnología, para luego contrastarlo con los resultados del autor del instrumento.

Las matemáticas, en particular la geometría, se ha visto permeada por las tecnologías, desde la década de los ochenta; Iglesias \& Ortiz (2018) proponen ver diferentes características o representaciones de un mismo objeto matemático que motive al estudiante aprovechando el interés que este tiene por el mundo digital. Entre tanto se propone una conexión entre Tecnologías de la Información y la Comunicación (TIC) y motivación en el aprendizaje de las matemáticas (Maggiolini, 2013; Villamizar, Rincón \& Vergel, 2018).

La motivación académica es importante para el desarrollo y entrega de actividades por parte del estudiante y de manera especial cuando se relaciona la tecnología, esto lo pudo comprobar Peña \& Aldana (2014), al desarrollar una propuesta tecnológica y contrastada con un diagnóstico inicial. Es así, que las Tecnologías de la Información y la Comunicación (TIC) promueven la 
motivación en los estudiantes y esto recae en mejoras del aprendizaje y aprovechamiento del tiempo libre (Delgado, Arrieta \& Riveros, 2009), además de las TIC, se debe tener presente el aspecto emocional del estudiante como lo pudo agregar en su investigación Cueli, González, Rodriguez, Núñez \& González (2018).

En su trabajo estadístico sobre las variables motivacionales de los estudiantes, Garrote, Garrote \& Jiménez (2016) encontraron que de manera principal estas son: metas intrínsecas, el valor de la tarea, la autoeficacia, las metas extrínsecas, las creencias de control y la ansiedad. Dichas variables cambian dependiendo el grado de escolaridad y según Núñez \& González, (2017) la motivación intrínseca se va perdiendo mientras que la extrínseca se refuerza, esto se nivela sobre los 16 años de edad.

El estudio de la función cuadrática según los estándares del Ministerio de Educación Nacional (Ministerio de Educación Nacional, 2006), sugieren ser abordado en el ciclo octavo-noveno; para el desarrollo de la investigación se relaciona en el noveno grado. El abordaje de este objeto matemático mediado por las tecnologías (GeoGebraß), permite una mayor aprehensión de sus características, comparadas con textos planos (Allan, Parra \& Martins, 2017; Aya, Echeverry \& Samper, 2016; García, Parra, Martínez \& Sóstenes, 2019; Gómez, Guirette \& Morales, 2017; Guachun \& Mora, 2019).

Se propone entonces, en el presente artículo indagar las variables motivacionales del estudiante en el estudio de la función cuadrática, de la forma $f(x)=a x^{2}+b x+c$, mediada por el software de geometría dinámica GeoGebraß).

El abordaje de la función cuadrática de la forma $f(x)=a x^{2}+b x+c$ realizado por Gómez et al. (2017) en el que propusieron algunas actividades mediadas por el software GeoGebraß, logró comprobar que las características visuales, simbólicas de este objeto matemático se ven más explícitas en el software que en los libros de texto. Aunque hace falta profundizar en esta herramienta para determinar con certeza si se puede trabajar solamente con esta herramienta (Azzolina, Saldivia \& Maglione, 2019).

La geometría dinámica permite la construcción y comprobación de argumentos por medio de la visualización y experimentación, pues ésta se caracteriza por permitir la manipulación de los objetos matemáticos sin que pierdan sus atributos esenciales (Cruz \& Mantica, 2017), lo cual brinda ventajas que no se encontrarán en lugares estáticos (Sua, 2019). Por su parte, Costa \& Sombra del Río (2019), aseguran que el software sí permite la aprehensión de conceptos (como la función) si al momento de su manipulación, el estudiante cuenta con los saberes asociados y un equipamiento praxeológico mínimo.

Una ventaja sobre el aprendizaje que cabe mencionar en la geometría dinámica, es que ésta no es lineal, sino que se da en la medida del avance de una situación problema, descubrimiento de propiedades y orientaciones del docente, en el que se hace necesario la observación y las deducciones que se deben justificar a medida que se descubren en el proceso (Larios, Pino \& 
González, 2017). Es muy común la metodología de resolución de problemas mediante la manipulación de un software, pues se ponen en juego distintos conocimientos y habilidades instrumentales que el docente debe manejar y el estudiante experimenta (Arteaga, Medina \& Del sol, 2019). Es importante que este proceso de construcción esté acompañado, regulado y evaluado para que realmente se dé un avance cognitivo en el aprendizaje cuando se utiliza algún tipo de software (Sua, 2019), agregando que, la institucionalización es de vital importancia para el cierre de procesos y abordajes, con el uso de los mismos (Flórez, 2016).

El artículo de Cruz \& Mantica (2017) titulado: El uso del software de geometría dinámica en la formulación y validación de conjeturas, los autores pudieron constatar que, los docentes deben colocar problemas iniciales para que los estudiantes exploren por medio de algún software y que ellos construyan propiedades de algún objeto matemático. No solo en la comprobación matemática se han hecho estudios de la geometría dinámica sino también en la argumentación, Larios et al. (2017), en su trabajo: esquemas argumentativos de estudiantes de secundaria en ambientes de geometría dinámica, muestra cómo el software permite desde la observación construir y comprobar hipótesis, aunque no siempre los estudiantes logran resolver problemas de aplicación por medio de la utilización del software de Geometría (Linares, 2020).

\section{Metodología}

La metodología de esta investigación es de tipo mixta, porque cumple con las características propuestas por Hernández, Fernández \& Baptista (2014); McMillan \& Schumacher (2005); Cortés \& Iglesias (2004). Desde el punto de vista cualitativo se apoya en el análisis interpretativo de una entrevista semiestructurada a un grupo focal (Flick, 2004). Lo anterior se complementa con un análisis cuantitativo, a partir de los registros obtenidos al aplicar el test CEAP-48 a los estudiantes, apoyado en la técnica de Análisis de Componentes Principales (ACP), para determinar las variables subyacentes (Dallas, 1998; Polo-Miranda, 2009; Walpole et al., 2007). La fiabilidad del instrumento se apoya en el Alfa de Cronbach (1951).

\section{Participantes}

Los participantes o la población de la investigación son estudiantes entre 13 y 16 años de un colegio de carácter privado en el Sur de Bogotá (Colombia), de estrato social tres. En total son ochenta y seis (86) adolescentes, en su mayoría hijos de personas dueñas de pequeñas empresas o negocios de emprendimiento como calzado o panadería. El colegio cuenta con dos salas de sistemas así como con algunas otras herramientas tecnológicas. Sin embargo, el escenario de la investigación se dio de manera virtual o presencialidad remota, por la realidad mundial del COVID19. Al inicio de la investigación los estudiantes manifestaron su interés por aprender de manera lúdica y social, construyendo y aprendiendo con el otro de forma colaborativa, por medio de las Tecnologías de la Información y la Comunicación (TIC). 


\section{Diseño metodológico}

El diseño metodológico se basa en el propuesto por Hernández et al. (2014) en el que, hay un grupo al cual se le aplica un test antes y después de la secuencia didáctica. En la figura 1 se indica la organización del diseño metodológico.

Figura 1. Diseño metodológico en el que se identifica el grupo a intervenir [G], la secuencia didáctica $[X]$, el pretest y el postest que corresponde a la aplicación de la escala de Motivación del Aprendizaje y Estilos Atribucionales 48 (CEAP-48) $\left[O_{1}\right]$ y la entrevista semiestructurada $\left[O_{2}\right]$.

$$
G \rightarrow O_{1} \rightarrow X \rightarrow O_{1} \rightarrow O_{2}
$$

Fuente: Los autores (2021)

\section{Instrumentos}

Los instrumentos utilizados en la investigación son tres principalmente: test de motivación CEAP-48 de Barca et al. (2005); Secuencia Didáctica (SD) y entrevista semiestructurada. El instrumento tiene una validez diacrónica, en el entendido que ha sido utilizado por diversas investigaciones en diferentes contextos, manteniendo unas medidas de fiabilidad entre buenas y excelentes.

\section{Test Motivacional CEAP-48}

El test de motivación CEAP-48 de Barca et al. (2005) busca entender los estilos motivacionales y atribucionales de una población, cuenta con dos escalas: SEMAP-01, llamada escala de motivación y escala SEAT-01, que reúne las características atribucionales del éxito académico; en total el test cuenta con cuarenta y ocho (48) ítems que se distribuyen de forma equitativa, medidos con una escala de Likert.

Las categorías propias de la escala SEMAP-01 son: motivación intrínseca/profunda, la cual describe la motivación interna del estudiante; motivación extrínseca/ motivación de aprendizaje y rendimiento, que encierra el motor que viene de fuera del estudiante para realizar una actividad; motivación superficial, la cual reúne todo lo que hace el estudiante para ser aceptado en un lugar o simplemente cumplir para aprobar un aprendizaje. En la tabla 1 se muestra la relación de los ítems (Anexo 1) que componen cada categoría en esta escala de motivación:

\section{Tabla 1.}

Categorías de la escala SEMAP-01 del test CEAP-48

\begin{tabular}{l|l}
\multicolumn{1}{c|}{ Categoría } & \multicolumn{1}{c}{ Ítems } \\
\hline Motivación intrínseca/ profunda & $2,5,8,11,14,17,20,7$ \\
\hline $\begin{array}{l}\text { Motivación extrínseca/ } \\
\text { Motivación de Aprendizaje y rendimiento }\end{array}$ & $3,12,13,15,18,21,22,23$ \\
\hline Motivación Superficial & $1,4,6,9,10,16,19,24$ \\
\hline
\end{tabular}

Fuente: Barca et al. (2005) 
En la escala SEAT-01, se cuenta con las categorías propias del test: estilo atribucional de metas de Rendimiento, de aprendizaje y esfuerzo (MRMAP/AE), relacionado con el esfuerzo personal que hace el estudiante por aprender, ya sea desde diferentes ámbitos de su vida; estilo atribucional a la suerte del bajo rendimiento escolar (SBRA/ARA), que implica relacionar el fracaso académico con la suerte o el azar; estilo atribucional mixto: al profesorado, al esfuerzo, a la Capacidad, a la Facilidad materias (EATM), que relaciona el éxito académico como algo que está en manos del docente y depende de la facilidad de los aprendizajes; estilo atribucional a la facilidad de las materias del bajo rendimiento académico (FM/BRA), que envuelve el hecho de pérdida académica por la falta de facilidad de las asignaturas; estilo atribucional a la baja capacidad del bajo rendimiento y al escaso esfuerzo (BCAE/BRA), que encierra el poco esfuerzo y la poca capacidad del estudiante en un aprendizaje específico. En la tabla 2 se muestra la relación de los ítems (Anexo 2) que componen cada categoría en esta escala de estilos atribuciones SEAT-01.

Tabla 2.

Categorías de la escala Seat-01 del test CEAP-48

\begin{tabular}{|c|c|}
\hline Categoría & Ítems \\
\hline Estilo atribucional de Metas de Rendimiento, de Aprendizaje y esfuerzo (MRMAP/AE) & $\begin{array}{l}15,21,16,7, \\
8,1,14,9\end{array}$ \\
\hline Estilo atribucional a la suerte del bajo rendimiento escolar (SBRA/ARA) & $23,19,12,5$ \\
\hline Estilo atribucional mixto: al Profesorado, al esfuerzo, a la Capacidad, a la Facilidad materias (EATM) & $\begin{array}{l}13,4,2,17,6, \\
10\end{array}$ \\
\hline Estilo atribucional a la facilidad de las materias del bajo rendimiento académico (FM/BRA) & 20,24 \\
\hline Estilo atribucional a la baja capacidad del bajo rendimiento y al escaso esfuerzo (BCAE/BRA) & $22,3,11,18$ \\
\hline
\end{tabular}

Fuente: Barca et al. (2005)

El test de Barca et al. (2005) tiene una validez diacrónica en el tiempo, siendo aceptado y adaptado en varias investigaciones (Apolinario, Rosales \& Condor, 2018; Barca, Do Nascimento, Brenlla, Porto \& Barca, 2008; Díaz, 2018; Fernández, 2009; Hernández, Martín, Lorite \& Granados, 2018; Rodriguez, 2017; Ruíz \& Bocanegra, 2019), mostrando resultados similares en su consistencia de Alfa de Cronbach, en relación con las categorías propias del instrumento.

\section{Secuencia Didáctica (SD)}

La secuencia didáctica (SD) fue construida con la idea de relacionar la motivación de los estudiantes, el software GeoGebra $\Re$ y el estudio de la función cuadrática; fue evaluada por cuatro pares y expertos bajo la validez de Aiken (Robles, 2018), obteniendo una aceptación y aprobación mayor al 70\%. Los criterios propuestos para revisar la estructura y validez del instrumento se muestran en la tabla 3. Los indicadores describen 1, cuando la SD NO cumple en absoluto y 5 cuando la SD cumple totalmente. 
Tabla 3.

Resultados de las categorías de evaluación del instrumento luego de aplicarle el test de Aiken

\begin{tabular}{|c|c|c|c|c|c|c|}
\hline \multirow{2}{*}{ INDICADOR } & \multicolumn{6}{|c|}{ VALIDADOR } \\
\hline & 1 & 2 & 3 & 4 & 5 & Promedio \\
\hline $\begin{array}{l}\text { En la secuencia didáctica se evidencian los principios del } \\
\text { aprendizaje basado en la colaboración. }\end{array}$ & 0,25 & 0,75 & 0,75 & 1,00 & 0,75 & 0,70 \\
\hline $\begin{array}{l}\text { Es coherente la secuencia didáctica con sus objetivos de } \\
\text { aprendizaje expuestos. }\end{array}$ & 0,50 & 1,00 & 1,00 & 1,00 & 0,75 & 0,85 \\
\hline $\begin{array}{l}\text { La organización conceptual de los contenidos presentados } \\
\text { en la secuencia didáctica es pertinente para los estudiantes } \\
\text { de grado noveno. }\end{array}$ & 0,75 & 0,75 & 1,00 & 1,00 & 1,00 & 0,90 \\
\hline $\begin{array}{l}\text { Los conceptos presentes en la secuencia didáctica son } \\
\text { apropiados para los estudiantes de grado noveno. }\end{array}$ & 0,75 & 0,75 & 1,00 & 1,00 & 1,00 & 0,90 \\
\hline $\begin{array}{l}\text { Las actividades presentes en la secuencia didáctica son } \\
\text { convenientes para los estudiantes de grado noveno. }\end{array}$ & 0,75 & 0,75 & 1,00 & 1,00 & 0,50 & 0,80 \\
\hline $\begin{array}{l}\text { El lenguaje utilizado en la secuencia didáctica es apropiado } \\
\text { para estudiante de grado noveno. }\end{array}$ & 0,75 & 0,75 & 0,75 & 1,00 & 0,75 & 0,80 \\
\hline $\begin{array}{l}\text { Es adecuado el uso de GeoGebrå en la secuencia } \\
\text { didáctica. }\end{array}$ & 1,00 & 0,50 & 0,50 & 1,00 & 0,50 & 0,70 \\
\hline
\end{tabular}

Fuente: Los Autores (2021)

Como se denotó con anterioridad, los resultados de la validación se analizan usando el coeficiente de validez V de Aiken (Robles, 2018), en el que se plantean los valores entre 0 y 1. Dado que los valores en la escala seleccionada, se encuentran entre 1 y 5 , siendo 1 como poco pertinente y 5 totalmente pertinente, éstos se deben convertir a la numeración de 0 a 1 (como lo relaciona el autor); para este caso la conversión será de la siguiente forma:

Restar 1 a los valores de la escala, quedando

$$
5-1=4 ; 4-1=3 ; 3-1=2 ; 2-1=1 ; 1-1=0
$$

Así los valores de la escala ahora son $0,1,2,3$ y 4, donde 1 es el mayor valor, por lo tanto 1 se divide en 4 , dando como resultado 0.25 , luego este valor se multiplica por cada número de la siguiente forma: $0 \times 0,25=0,00 ; 1 \times 0,25=0,25 ; 2 \times 0,25=0,50 ; 3 \times 0,25=0,75 ; 4 \times 0,25=1,00$.

Teniendo los valores entre 0,00 y 1,00 se reemplazan los datos obtenidos de los validadores (pares y expertos) como se muestra en la tabla 3. Los valores del índice de Aiken, en promedio, están por arriba de 0,70 por lo que, por parte del equipo evaluador, es posible afirmar que hay validez en cada uno de los criterios para la secuencia didáctica. 


\section{Figura 2.} sesión.

Pantalla de GeoGebra \& en el que se observa una parte de la actividad desarrollada en la primera

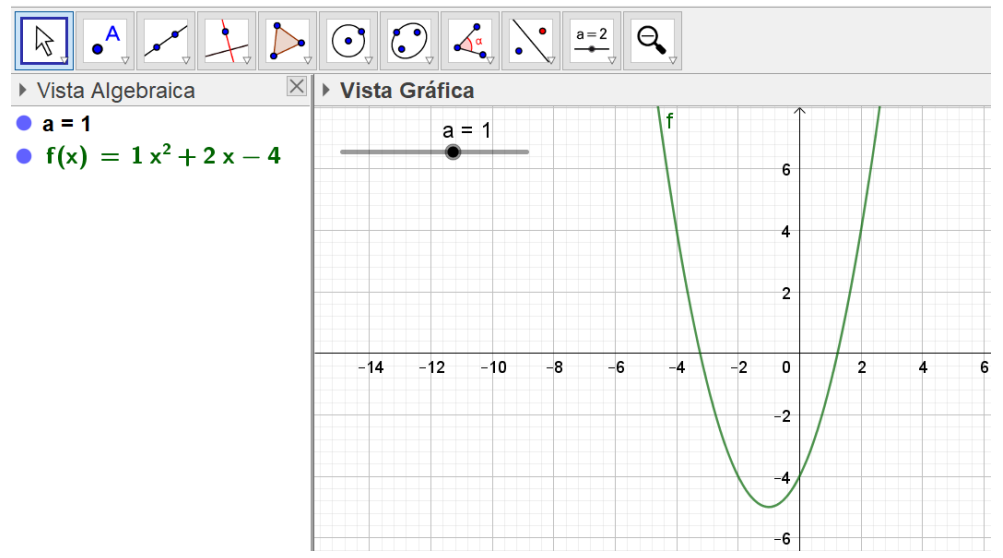

Fuente: Los Autores (2021)

La secuencia didáctica se desarrolló en cuatro (4) sesiones de clase; la primera "conociendo la función cuadrática y su parámetro a" (parte de la actividad como se observa en la figura 2), la segunda "caracterizando los parámetros b y c de la función cuadrática", la tercera "investigando aprendo" orientada al estudio de los puntos de corte de la función cuadrática y la cuarta "¿esto para qué me sirve?" dedicada que el estudiante interactúe con situaciones problema de aplicación en diferentes contextos.

\section{Entrevista grupo focal}

Como parte de la recolección de la información, se realizó la entrevista semiestructurada de los grupos focales, a seis (6) estudiantes de diferentes grupos de trabajo, escogidos de forma aleatoria; dicha entrevista se transcribió y categorizó en el software Atlas Tiß, asumiendo las mismas categorías de las Variables Subyacentes (VS), del test de motivación académica.

A continuación se presentan las preguntas base de la entrevista semiestructurada:

1. ¿Qué fue lo que más le llamó la atención del trabajo colaborativo en la actividad? ¿Por qué?

2. ¿Cuáles fueron los factores o cosas que generaron motivación en el aprendizaje de la función cuadrática?

3. ¿Qué lo motiva para aprender en cualquier aprendizaje? ¿Y en matemáticas?

4. ¿Qué elementos encuentra en común entre matemáticas, colaboración, motivación y TIC?

5. ¿Qué genera motivación en usted cuando tiene contacto con las TIC? 
6. ¿Qué rescata o le parece muy importante del software GeoGebra®? ¿Por qué?

7. ¿Qué no le gustó de la experiencia en el aprendizaje de la función cuadrática?

8. ¿Es importante lo que aprendió en todo el proceso? ¿Por qué?

9. ¿Cómo se sintió en todo el proceso de aprendizaje de la función cuadrática? ¿Por qué?

10. Las notas, el trabajo colaborativo, las TIC ¿̇lo motivan en el aprendizaje de las matemáticas? ¿Por qué?

\section{Procesamiento de la información}

En el análisis de información, se utilizaron los siguientes software: SPSS $®$, usado para relacionar el método de Análisis de Componentes Principales (ACP) y el Alfa de Cronbach;

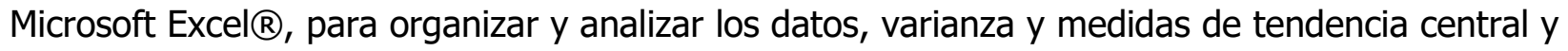
Atlas Tiß, para asociar categorías a la transcripción escrita de la entrevista semiestructurada.

Sobre el Alfa de Cronbach, se puede afirmar que éste muestra la consistencia interna que estima la fiabilidad de la agrupación de los ítems de un instrumento que se espera sean congruentes a una misma categoría, estos análisis se dan por medio de las varianzas en los Análisis de Componentes principales, que surgen de estimar las respuestas a los ítems que están en la escala de Likert. Se precisa que el alfa va de 0,000 a 1,000; entre más cerca de 1,000, mayor es la consistencia interna entre los ítems asociados (Frías-Navarro, 2020).

Los datos estadísticos obtenidos permiten realizar inferencias de la varianza, la estadística descriptiva, la media y desviación estándar de los datos agrupados en categorías. El Análisis de Componentes Principales (ACP) se utiliza como un mecanismo de reducción de variables. Se tiene en cuenta que la primera componente es la agrupación que tenga mayor varianza explicada. Para esta investigación las variables (entiéndase como cada ítem en cada un cuestionario) que se agrupan, se les denomina variables subyacentes.

En cuanto a la entrevista semiestructurada que se realizó al grupo focal, se transcribió de forma manual para luego procesarse en el software Atlas $\mathrm{Ti}$, se conformaron redes de los códigos ampliando el panorama de relación entre las categorías de motivación antes y después de la aplicación de la secuencia didáctica. 


\section{Resultados y discusión}

Los resultados obtenidos de la población de la presente investigación fueron utilizados y analizados con el software SPSS $\Re$, de acuerdo a la escala y categoría propia del instrumento CEAP-48, demostrando que tanto en el pretest como en el postest, los resultados son similares, variando por algunas centésimas, pero estando dentro del mismo margen de confiabilidad (FríasNavarro, 2020), lo que resulta interesante porque da cuenta de dos cosas: que el instrumento es válido y consistente para esta investigación y, que los resultados de la escala SEMAP-01 y SEAT01 son diacrónicos en el tiempo, de manera independiente si hay o no presencia de la tecnología.

Después de aplicar la técnica ACP, que arroja las variables linealmente independientes, emergieron cuatro (4) variables subyacentes para el pretest y cinco (5) variables subyacentes para el postest, las que se interpretan y discuten en las líneas siguientes. En la figura 3 se sintetizan los resultados de la reducción de variables, en la que se identifican el Alfa de Cronbach global que se puede calificar como bueno (Frías-Navarro, 2020).

Sobre la categoría de examen como motivación en el pretest, se puede ver en los ítems agrupados, que el examen es un factor de motivación extrínseca e influye en el gusto del niño, y que no es agradable, pues existen estudios como el de Bausela (2005) que asevera que la prueba trae ansiedad y miedo por perder el aprendizaje. De igual forma, los resultados del examen crean estatus de rechazo o discriminación (Csóti, 2011) y en algunos casos la figura de fracasados (Romero, Utrilla, Utrilla, 2014).

\section{Figura 3.}

Variables subyacentes obtenidas del Análisis de Componentes Principales de la sub escala de motivación académica SEMAP-01, antes y después de la aplicación de la Secuencia Didáctica (SD), en el que se identifica el alfa de Cronbach [ $\alpha$ ], varianza explicada $\left[\sigma^{2}\right]$ y las afirmaciones que la componen.

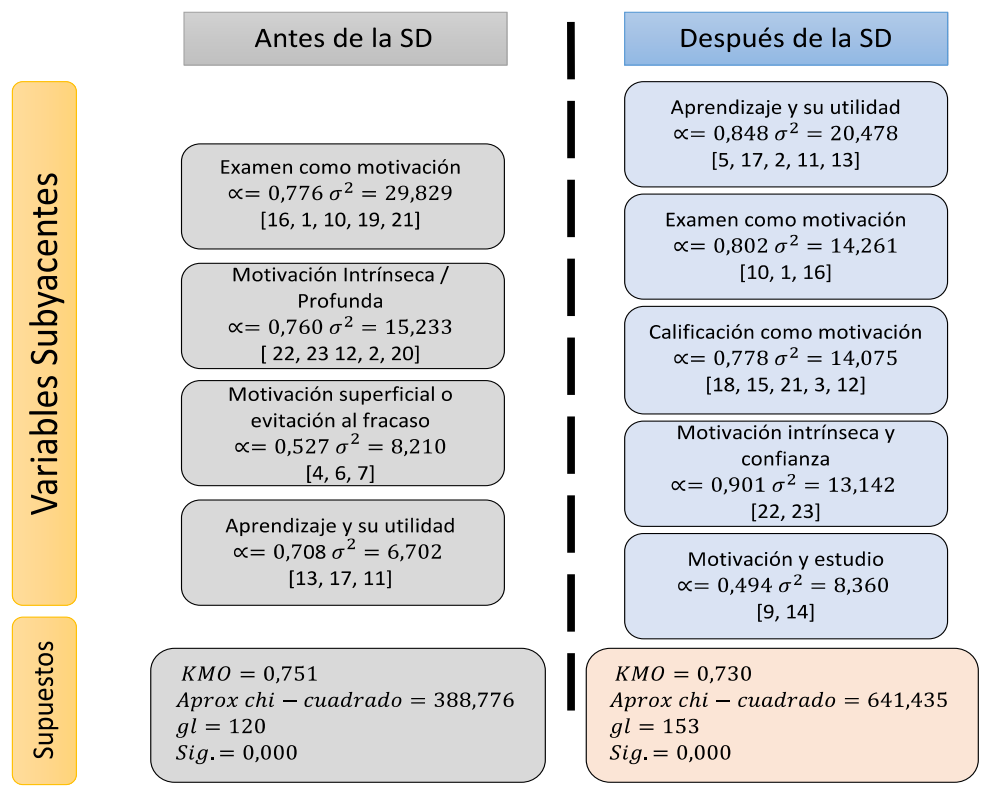

Fuente: Los Autores (2021) 
En la figura 3 se puede evidenciar que la categoría de examen como motivación, se mantuvo tanto en el pretest como en el postest, pasando de un Alfa 0,776 a 0,802, lo cual da a entender que los ítems de la categoría se relacionan de forma más consistente, pasando de ser aceptable a bueno según Frías-Navarro (2020), por lo que la evaluación entendida desde el examen se relaciona con la motivación, indistintamente si hay presencia o no de la tecnología, que para este caso se evidenció en la utilización de GeoGebraß. Pedrosa (2015) explica que dicha relación, al parecer, es inversa porque genera miedo o vergüenza y produce reacciones negativas en los estudiantes; por su parte A1 (estudiante que participó en la entrevista semiestructurada) manifiesta que"...me estresaba y empezaba a llorar porque no sabía cómo hacerlo...". Lo permite inferir que el examen genera emociones negativas en el estudiante, al no poder resolver en primera instancia lo que éste le presenta.

La motivación profunda (motivación intrínseca) en el pretest muestra el valor de la inquietud intelectual ligada a la competitividad y autoestima, reflejados en el deseo de entender lo que se está estudiando o adquiriendo (Elizondo et al., 2018). El estudiante A3, dice que la motivación "...es también como la responsabilidad, como las ganas de entregar el trabajo...", la responsabilidad por cumplir, viene con el sujeto y no tanto por estímulo externo. La eficacia se puede ver entre líneas de los ítems agrupados, lo cual define la confianza que tiene cada individuo de sus propias capacidades para realizar una tarea y que entre mayor sea la autoeficacia, existe una mayor motivación intrínseca (Rossi, Trevisol, Santos, Dapieve \& Hohendorff, 2020).

La motivación intrínseca estuvo presente en el pretest con un alfa de 0,760 y en el postest con un alfa de 0,901 y según Frías-Navarro (2020) pasó de ser buena a excelente. Se conjetura que la motivación intrínseca es relevante para los estudiantes, de forma independiente a la presencia de la tecnología en las actividades académicas.

La motivación superficial o de evitación al fracaso, parece relacionarse con la motivación intrínseca que trasciende a la escuela, como la exclusión de un contexto social según lo expresan Hernández \& Tort (2009) y para este caso podría ser su entorno social o familiar.

La variable subyacente motivación superficial o evitación al fracaso estuvo presente antes de aplicar la SD, en tanto que después de aplicarla, no se refleja en las variables. Lo anterior podría tener su origen en la presencia de la tecnología, a través de GeoGebra ${ }^{\circledR}$, lo que se constituye en un indicio de la existencia de una relación entre el gusto por hacer las cosas y la incorporación de la tecnología. Sin embargo, es necesario precisar que el presente estudio no da suficiente evidencia del anterior análisis por lo que futuras investigaciones podrán develar la existencia 0 no de la relación.

En el pretest de la motivación académica, el aprendizaje y su utilidad son de vital importancia para el estudiante logre relacionar lo que estudia, con lo que desea desempeñar en su futuro laboral (Moreira, 2019), que pueda modificar su saber para adaptarlo al contexto actual de acuerdo a su experiencia con el mismo. Para el caso de los participantes de la investigación, algunos consideran que, existen elementos matemáticos que no sirven en su diario vivir, tal 
como lo indica el estudiante A2 cuando dice que "... yo digo que si sirve pero para el colegio, pero en la vida diaria ¿quién va a decir que el área de la piscina es $x+2$, no es que tengo una piscina de $x+2 x$ por $x+5$, la quiere comprar?...." Es por ello que el estudio de la función cuadrática debe ser considerada en profundidad en el currículo, para aportar cognitivamente a los estudiantes y éstos puedan comprender de forma real, lo que necesita aprender; lo anterior pone de presente de un lado la motivación y del otro lado la noción de variable (Ursini \& Trigueros, 2006, 1997).

La categoría de aprendizaje y su utilidad estuvo presente en el pretest con un alfa de 0,708 y en el postest con un alfa de 0,848, según Frías-Navarro (2020) pasó de ser aceptable a bueno. Para el estudiante resulta motivante ver la utilidad de lo que está estudiando, al parecer de forma indistinta si hay o no presencia de tecnología. Con la secuencia didáctica, los ítems de la categoría de aprendizaje y su utilidad parecen estar más relacionados y dar más información, pues se relacionaron de una manera más uniforme, manteniéndose en el tiempo.

La categoría de calificación o nota como motivación, refleja que la nota es un factor relevante en el estudiante para competir con otros, y que la comparación de las calificaciones es una forma de medición con respecto a los demás, independiente de su aprobación o no. Los participantes de la investigación recalcan que la nota si los motiva, lo que refiere que el pago y la satisfacción del estudiante se debe ver reflejada en una retribución numérica, y según Ochoa \& Moya (2019) esta motivación recae en el docente al enseñar bien o mal y concluye Lamas (2015) que la nota por parte del educador va de la mano con la atención que se preste al estudiante.

En cuanto a la escala SEAT-01 del instrumento, se encontró que las variables subyacentes antes de la aplicación de la secuencia didáctica (pretest) fueron: rendimiento escolar y la suerte (alfa de 0,832), esfuerzo y éxito (alfa de 0,733), capacidad y esfuerzo (alfa de 0,737), notas y facilidad por el tipo de inteligencia (alfa de 0,619).

Las variables subyacentes de la escala SEAT-01 de los estilos atribucionales después de la aplicación de la secuencia didáctica (postest) fueron: capacidad y esfuerzo (alfa de 0,845), éxito, profesorado y suerte (alfa de 0,785), facilidad y gusto (alfa de 0,694), capacidad (alfa de 0,570). En la figura 4 se relacionan los resultados del pretest y el postest de la escala SEAT-01. 
Figura 4.

Variables subyacentes obtenidas del análisis de componentes principales de la sub escala de atributos causales SEAT-01 antes y después de la secuencia didáctica (alfa de Cronbach [ $\alpha$ ], Varinza explicada $\left[\sigma^{2}\right]$, afirmaciones que la componen).

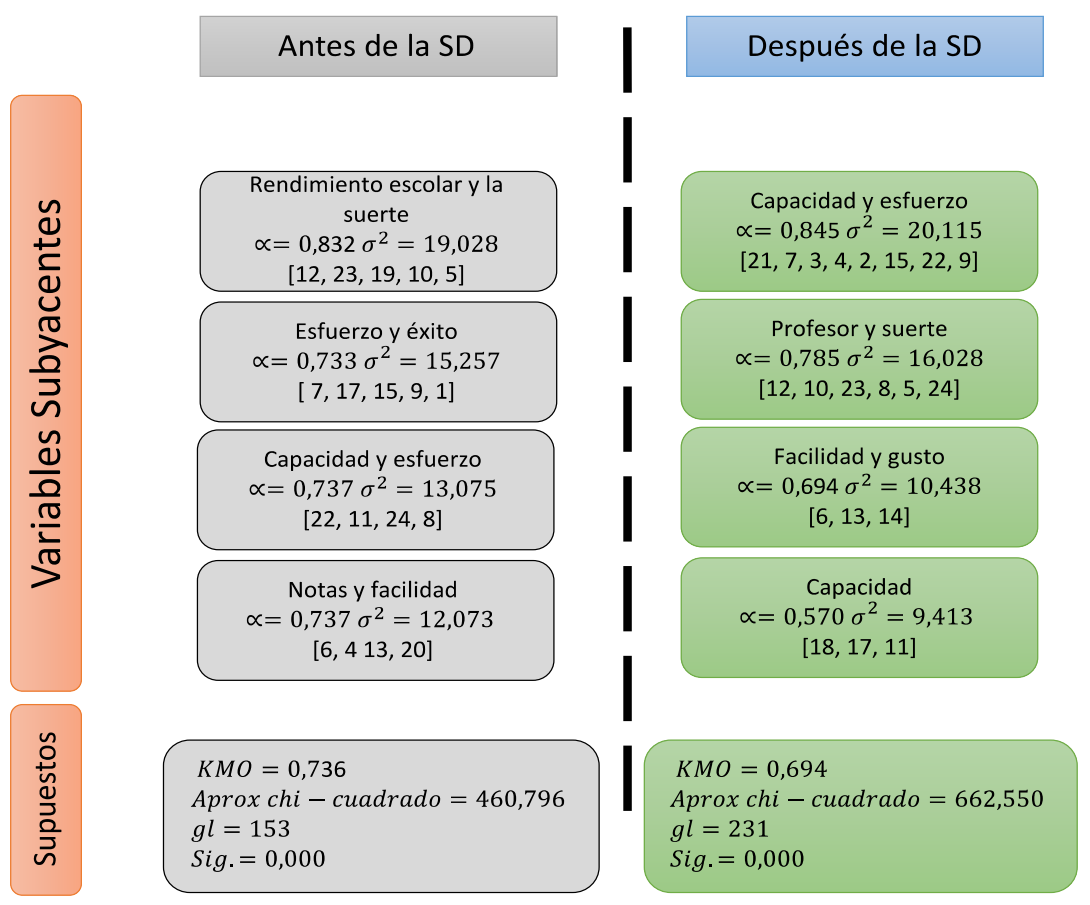

Fuente: Los Autores (2021)

En el pretest, las categorías de: esfuerzo y éxito; capacidad y esfuerzo tienen un alfa aceptable (Frías-Navarro, 2020), las notas y facilidad por el tipo de inteligencia, tienen un alfa cuestionable (Frías-Navarro, 2020), lo que pone en debate la veracidad de los ítems agrupados por el software SPSSR, al relacionar las notas directamente con el tipo de inteligencia del estudiante.

En los estilos atribucionales se puede ver que, la categoría de capacidad y esfuerzo se mantuvo de 0,733 a 0,845 lo que según Frías-Navarro (2020) pasando de aceptable a bueno en su confiabilidad del Cronbach, lo que indica que los estudiantes atribuyen el esfuerzo y la capacidad al éxito académico de cada uno, de manera independiente si existe o no presencia de tecnología. Este mismo razonamiento se puede establecer en las otras categorías halladas por el ACP en la escala SEAT-01 del postest, pues lo atribucional al éxito académico no varía con una intervención pedagógica en la que haya influencia de las TIC.

La categoría de rendimiento escolar y la suerte, propone que el éxito académico tiene una relación directa con la suerte y el azar, lo que contrasta con otras investigaciones (Barca et al., 2005; Fernández, 2009) reforzando que la aprobación o no del curso depende del profesorado. 
El esfuerzo y el éxito involucran la capacidad del estudiante para llegar a obtener mejores resultados, pues el éxito académico se relaciona de forma directa con el esfuerzo (Lagos, Inglés, Ossa, Gonzálvez, Vicent \& García, 2016) pero ese aprender debe venir tanto por parte del estudiante como del docente (Fernández, 2009).

La capacidad y el esfuerzo van de la mano de lo fácil o difícil que resulte un aprendizaje, lo que recae en la capacidad intelectual del estudiante que lo lleva a una reprobación o fracaso académico (Lagos et al., 2016). La suma de esfuerzos crea capacidad, al parecer la capacidad no solamente es individual sino que es resultado de una construcción colectiva:

...pues, que cada una del grupo pues tenía como una idea de... digamos tenía una idea creo que fue en el último, en el de los tapabocas, cada una tenía una idea diferente entonces pues como que pues uno mira distintas como podría decirse soluciones pues para hacer un problema y ya... (Estudiante E2). En resumen, la colaboración es una oportunidad para crear una capacidad colectiva, que permite aprobar el curso. Así mismo, el éxito académico depende en parte del tipo de inteligencia del sujeto (Gardner, 1983) y la metodología del docente.

\section{Conclusiones}

Las categorías del inventario CEAP-48 test de motivación propuesto por Barca et al. (2005), arrojaron alfas similares al ser comparadas y analizadas con el pretest y postest de la investigación, lo que lleva a considerar dos cosas: indistintamente del tiempo, el instrumento mantiene indicadores de consistencia entre buenos y excelentes (Frías-Navarro, 2020); y al pasar quince (15) años hasta llegar al año 2020, la motivación se puede seguir describiendo por las categorías de motivación intrínseca o profunda, motivación de aprendizaje y rendimiento o extrínseca, motivación superficial. Por lo anterior, el instrumento tiene una validez diacrónica en el tiempo y se puede seguir utilizando para futuras investigaciones.

La categoría de examen como motivación estuvo presente en el pretest y en el postest, lo que muestra, que indistintamente del uso de la tecnología (para esta investigación GeoGebraß) el examen hace parte de la motivación académica extrínseca del estudiante, así como una influencia negativa en sus emociones como la ansiedad, miedo o rechazo (Suárez, Hurradi \& Pulido, 2020).

Al parecer el aprendizaje y su utilidad es de sumo interés en los participantes de la investigación y existe además preocupación por su futuro laboral desde la escuela, se puede denotar que esta categoría en la escala SEMAP-01 estuvo presente en el pretest y en el postest. Es aquí que recae la importancia que el estudiante utilice el conocimiento que adquiere en su diario cotidiano, lo que concuerda con lo expuesto por Moreira (2019).

Es interesante cómo la calificación o nota como motivación (alfa de 0,778 ) tiene cierta relevancia, pues ésta es importante para la aprobación del aprendizaje, lo que se constituye en 
un obstáculo o barrera para la motivación intrínseca, pues si no está este estímulo, el estudiante desiste de emprender el trabajo académico.

El éxito académico depende en parte, y de forma independiente a la tecnología, por el azar, la suerte y los profesores, pues se analizó que esta categoría estuvo presente tanto en el pretest como en el postest de la escala SEAT-01. De la misma forma sucedió con la categoría facilidad y gusto en el que, sin importar que exista presencia de la tecnología, se relaciona directamente con los resultados de los aprendizajes.

A manera de reflexión final, el uso de GeoGebra $®$ desde el punto de vista de los estudiantes, al parecer permitió reconocer diferentes representaciones de la función cuadrática, lo que concuerda con lo expresado por Osorio, Palacios \& Vallejo (2019) y por lo señalado por Gómez et al. (2017) en el que, con este software se puede apreciar de una forma clara las características de la función cuadrática.

\section{Referencias}

Allan, C., Parra, S., \& Martins, A. (2017). Objetos de Aprendizaje para la Interpretación Geométrica de Métodos Numéricos: Uso de GeoGebra. Revista Iberoamericana de Tecnología en Educación y Educación en Tecnología, 20(1), 51-56.

Apolinario, L., Rosales, I., \& Condor, A. (2018). Motivación e inteligencia emocional en las estudiantes de Tecnología del Vestido de la Universidad Nacional de Educación Enrique Guzmán y Valle, 2016. [Tesis de pregrado, Universidad Nacional de Educación Enrique Guzmán y Valle].

Arteaga, E., Medina, J., \& Del sol, J. (2019). El Geogebra: una herramienta tecnológica para aprender matemática en la secundaria básica haciendo matemática. Conrado, 15(70), 102-108. http://conrado.ucf.edu.cu/index.php/conrado

Aya, O., Echeverry, A., \& Samper, C. (2016). ¿Es el cuadrado un rectángulo? Sophia, 12(1), 139158. https://doi.org/10.18634/sophiaj.12v.1i.451

Azzolina, Y. A., Saldivia, F., \& Maglione, D. (2019). Un posible abordaje para enseñar función cuadrática en un ambiente tecnológico. Informes Científicos Técnicos - UNPA, 11(3), 1532. https://doi.org/10.22305/ict-unpa.v11.n3.795

Barca, A., Do Nascimento, S. a., Brenlla, J. C., Porto, A. M., \& Barca, E. (2008). Motivacion y aprendizaje en el alumnado de Educación Secundaria y rendimiento académico: un análisis desde la diversidad e inclusión educativa. Amazônica Revista de Psicopedagogia, 1(1), 957.

Barca, A., Porto, A., Santorum, R., Brenlla, J., Morán, H., \& Barca, E. (2005). La escala CEAP48: 
un instrumento de evaluación de la motivación académica y atribuciones causales para el alumnado de enseñanza secundaria y universitaria de Galicia. Revista de Psicología y Educación, 1(2), 265-302.

Bausela, E. (2005). Evaluación E Intervención. Revista Venezolana de Educación, 9(31), 553558. https://doi.org/10.1504/IJCAT.2008.022430

Carrillo, M., Padilla, J., Rosero, T., \& Villagómez, M. (2009). La motivación y el aprendizaje. A/teridad, 4(2), 20-32. https://doi.org/10.17163/alt.v4n2.2009.03

Cortés, M., \& Iglesias, M. (2004). Generalidades sobre Metodología de la Investigación. https://www.unacar.mx/contenido/gaceta/ediciones/metodologia_investigacion.pdf

Costa, V., \& Sombra del Rio, L. (2019). Aportes de la Geometría Dinámica al estudio de la noción de función a partir de un problema geométrico: un análisis praxeológico. Bolema: Boletim de Educação Matemática, 33(63), 67-87. https://doi.org/10.1590/1980-4415v33n63a04

Cronbach, L. J. (1951). Coefficient alpha and the internal structure of tests. Psychometrika, 16(3), 297-334. https://doi.org/10.1007/BF02310555

Cruz, M., \& Mantica, A. M. (2017). El uso del software de geometría dinámica en la formulación y validación de conjeturas. Revista Iberoamericana de Educación, 51(1), 69-82.

Csóti, M. (2011). Fobia escolar, ataques de pánico y ansiedad en niños. Lumen.

Cueli, M., González, P., Rodríguez, C., Núñez, J. C., \& González, J. (2018). Efecto de una herramienta hipermedia sobre las variables afectivo- motivacionales relacionadas con las matemáticas. Educación XX1, 21(1), 375-394. https://doi.org/10.5944/educXX1.12233

Dallas, J. (1998). Métodos multivariados aplicados al análisis de datos. International Thompson Editores.

Delgado, M., Arrieta, Xi., \& Riveros, V. (2009). Uso de las TIC en educación, una propuesta para su optimización. Omnia, 15(3), 58-77.

Díaz, J. (2018). Motivación académica y estilos atribucionales de los discentes del 1er. año de bachillerato de la Unidad Educativa Municipal "Eugenio Espejo", ubicada en la provincia de Pichincha en el cantón Quito, en la parroquia de Pusuquí, en el año lectivo 2017-2018. [Tesis de pregrado, Universidad Central de Ecuador].

Elizondo, A., Rodríguez, V., \& Rodríguez, I. (2018). La importancia de la emoción en el aprendizaje: Propuestas para mejorar la motivación de los estudiantes. Ventajas abiertas a la pedagogía universitaria, 15(29), 3-11.

Fernández, M. (2009). Motivación, aprendizaje y rendimiento académico en estudiantes de primer 
año de universidad en la República Dominicana. [Tesis doctoral, Universidad de Murcia].

Flick, U. (2004). Introducción a la Investigación Cualitativa. Ediciones Morata S. L.

Flórez, J. E. (2016). Enseñanza de la Simetría Axial utilizando situaciones a-didácticas y SGD, Car Metal como medio. http://funes.uniandes.edu.co/9863/

Frías-Navarro, D. (2020). Apuntes de consistencia de las puntuaciones de un instrumento de medida. Universidad de Valencia, España.

García, D., Parra, M., Martinez, H., \& Sostenes, S. (2019). Una propuesta didáctica para la enseñanza y el aprendizaje de la función lineal afín con el uso de la calculadora Classwiz. Acta Latinoamericana De Matemática Educativa, 32(1), 658-667.

Gardner, H. (1983). Multiple Intelligences. Paidós.

Garrote, D., Garrote, C., \& Jiménez, S. (2016). Factores influyentes en motivación y estrategias de aprendizaje en los alumnos de grado. REICE. Revista Iberoamericana sobre Calidad, Eficacia $y$ Cambio en Educación, 14(2), 31-44. https://doi.org/10.15366/reice2016.14.2.002

Gómez, A., Guirette, R., \& Morales, F. (2017). Propuesta para el tratamiento de interpretación global de la función cuadrática mediante el uso del software GeoGebra. Educación Matemática, 29(3), 189-224. https://doi.org/10.24844/em2903.07

Gómez, G., Rodríguez, C., \& Marín, J. (2020). La trascendencia de la Realidad Aumentada en la motivación estudiantil. Una revisión sistemática y meta-análisis. Alteridad, 15(1), 36-46. https://doi.org/10.17163/alt.v15n1.2020.03

Guachun, F., \& Mora, B. (2019). El software GeoGebra como resurso para la enseñanza de la función lineal: Una propuesta didáctica. Números, 101(1), 103-112.

Hernández, F., \& Tort, A. (2009). Cambiar la mirada sobre el fracaso escolar desde la relación de los jóvenes con el saber. Revista Iberoamericana de Educación, 49(8), 1-11. https://doi.org/10.35362/rie4981984

Hernández, L., Martín, C., Lorite, G., \& Granados, P. (2018). Rendimiento, motivación y satisfacción académica, ¿una relación de tres? Reidocrea, 7(9), 92-97.

Hernández, R., Fernández, C., \& Baptista, M. del P. (2014). Metodología de la investigación. MC GRAW HILL.

Iglesias, M., \& Ortiz, J. (2018). Usos del software de Geometría dinámica en la formación inicial de profesores de Matemáticas. Matemáticas, Educación y Sociedad, 1(2), 21-35. 
Lagos, N., Inglés, C., Ossa, C., Gonzálvez, C., Vicent, M., \& García, J. (2016). Relación entre atribuciones de éxito y fracaso académico y ansiedad escolar en estudiantes chilenos de educación secundaria. Psicología desde el caribe, 33(2), 146-157.

Lamas, H. (2015). Sobre el rendimiento escolar. Propósitos y representaciones, 3(1), 313-386.

Larios, V., Pino, L., \& González, N. (2017). Esquemas argumentativos de estudiantes de secundaria en ambientes de geometría dinámica. Avances de Investigación en Educación Matemática, 12(1), 39-57. https://doi.org/10.35763/aiem.v1i12.143

Linares, V. (2020). Efectos del uso de geeometría dinámica sobre el aprendizaje y la actitud haccia las matemáticas. Revista Internacional de Estudios En Educación, 2(1), 78-93. http://conrado.ucf.edu.cu/index.php/conrado

Maggiolini, L. M. (2013). Estrategias de motivación en una era digital: Teléfonos móviles y Facebook en el aula. Digital Education Review, 24(1), 83-97.

McMillan, J., \& Schumacher, S. (2005). Diseños y métodos de investigación cuantitativa. Investigación educativa.

Ministerio de Educación Nacional. (2006). Estándares Básicos de Competencias en Lenguaje, Matemáticas, Ciencias y Ciudadanas. Ministerio de Educación nacional.

Moreira, P. (2019). Las Tics en el aprendizaje significativo y su rol en el desarrollo cognitivo de los adolescentes. Rehuso, 4(2), 1-12. https://doi.org/10.33936/rehuso.v4i2.1845

Núñez, N., \& González, M. (2020). Formato Aula-Taller en primaria. Incidencia en la motivación y logros de aprendizaje de los estudiantes. Cuadernos de Investigación Educativa, 11(2), 133-155. https://doi.org/10.18861/cied.2020.11.2.2982

Núñez Soler, N. E., \& González, M. L. (2017). El formato aula taller y su incidencia sobre la motivación, el aprendizaje y el logro escolar de niños de nivel primario. Saberes $y$ prácticas. Revista de filosofía y educación, 2(1), 1-15.

Ochoa, L., \& Moya, C. (2019). La evaluación docente universitaria: retos y posibilidades. Folios, 49(1), 41-60. https://doi.org/10.17227/folios.49-9390

Osorio, K. T., Palacios, M., \& Vallejo, N. Y. (2019). Relación entre representación algebraica y gráfica de la función cuadrática mediada por Geogebra. [Tesis de pregrado, Universidad Cooperativa de Colombia].

Pedrosa, R. (2015). Reeducación cognitiva - emotiva en caso de ansiedad ante los exámenes. Revista Iberoamericana para la Investigación y el Desarrollo Educativo, 6(11), 1-17.

Peña, R., \& Aldana, E. (2014). El problema social y cultural de la población sorda en el aprendizaje 
de las matemáticas se minimiza con la intervención del profesor. Revista Latinoamericana de Etnomatemática, 7(2), 29-43.

Polo-Miranda, C. (2009). Estadística Multivariable. Ediciones UPC.

Robles, B. (2018). Índice de validez de contenido: Coeficiente V de Aiken. Pueblo continente, 29(1), 193-197.

Rodríguez, J. P. (2017). Motivación académica en el ciclo básico. Estudio realizado en el Instituto Nacional de educación Básica de telesecundaria aldea Chayen, San Marcos [Tesis de pregrado, Universidad de San Carlos]. https://doi.org/10.1177/0309133309346882

Romero, L., Utrilla, A., \& Utrilla, V. M. (2014). Las actitudes positivas y negativas de los estudiantes en el aprendizaje de las matemáticas, su impacto en la reprobación y la eficiencia terminal. $R a \quad$ Ximhai, 291-320. https://doi.org/10.35197/rx.10.03.e1.2014.20.Ir

Rossi, T., Trevisol, A., Santos, D., Dapieve, N., \& Hohendorff, J. Von. (2020). Autoeficacia general percibida y motivación para aprender en adolescentes de educación media. Acta Colombiana de Psicología, 23(1), 254-263. https://doi.org/http://doi.org/10.14718/ACP.2020.23.1.12

Ruíz, E., \& Bocanegra, E. (2019). Motivación en el aprendizaje de los estudiantes del sexto grado del nivel primaria de la I.E. N 0660 "Jorge Ruíz Veintemilla" San José de Sisa - 2016 [Tesis de pregrado, Universidad Nacional de San Martín de Tarapoto]. https://doi.org/10.1103/PhysRevA.76.032109

Sua, C. (2019). Saber suficiente no es suficiente: comportamientos metacognitivos al resolver problemas de demostración con el apoyo de la geometría dinámica. Tecné Episteme y Didaxis: TED, 45(1), 121-142. https://doi.org/10.17227/ted.num45-9838

Suárez, O., Hurtado, A., \& Pulido, O. (2020). Variables del estrés académico en estudiantes que cursan matemáticas en una facultad de ciencias administrativas y contables. Academia y Virtualidad, 13(1), 37-49. https://doi.org/10.18359/ravi.3713

Ursini, S., \& Trigueros, M. (2006). Mejoran la comprensión del concepto de variable cuando los estudiantes cursan matemáticas avanzadas? Education Matemática, 18(1), 5-38.

Ursini, S., \& Trigueros, M. (1997). Understanding of different uses of variable: A study with starting college students. 21st Conference of the International Group for the Psychology of Mathematics Education, 254-261.

Villamizar, F. Y., Rincón, O. L., \& Vergel, M. (2018). Diseño de escenarios virtuales para problemas de optimización a través de geometría dinámica. Revista Logos Ciencia \& Tecnología, 
10(2), 67-75. https://doi.org/10.22335/rlct.v10i2.571

Walpole, R., Myers, R., Myers, S., \& Ye, S. (2007). Probabilidad y estadística para ingeniería y ciencias. Editorial Pearson Educación S.A.

Zull, J. (2002). The art of changing the brain Enriching Teaching by Exploring the Biology of Learning. Stylus Publishing, LLC.

\section{Agradecimiento y apoyo financiero}

El presente artículo es resultado de un proyecto de investigación del grupo DIDATEC de la Maestría En Educación En Tecnología de la Universidad Distrital Francisco José de Caldas, Bogotá.

Agradecemos al Instituto San Ignacio de Loyola que permitió el desarrollo de la investigación en sus aulas de manera remota con los estudiantes de noveno grado.

Finalmente, los autores dedican este documento a todos los colegas y en general a las personas que han perdido la vida en medio de la pandemia del Covid-19. 


\section{Anexos}

\section{Anexo 1: preguntas en el test de motivación Semap-01}

A continuación los Ítems propuestos en la Subescala SEMAP-01 (Motivación del Aprendizaje) de la Escala CEAP48 propuesta por Barca et al. (2005):

1. Me desanimo fácilmente cuando obtengo una baja calificación

2. Me satisface estudiar porque siempre descubro algo nuevo

3. Pienso que es siempre importante obtener altas calificaciones

4. Reconozco que estudio para aprobar

5. Me gusta aprender cosas nuevas para profundizar después en ellas

6. Es muy importante para mí que los profesores y profesoras señalen exactamente lo que debemos hacer

7. Cuando no entiendo los contenidos o temas de las asignaturas es porque no me esfuerzo lo suficiente (En el artículo original no se alcanza a ver pero es rescatada por Beltrán, 2018)

8. Estudio a fondo los temas que me resultan interesantes

9. Me esfuerzo en el estudio porque mi familia me suele hacer regalos

10. A la hora de hacer los exámenes, tengo miedo de perder

11. Pienso que estudiar te ayuda a comprender mejor la vida y la sociedad

12. Me gusta competir para obtener las mejores calificaciones

13. Creo que estudiar facilita un mejor trabajo en el futuro

14. Cuando estudio aporto mi punto de vista o conocimientos propios

15. Lo importante para mí es conseguir buenas notas en todas las materias

16. Cuando hago los exámenes pienso que me van a salir peor que a mis compañeros/as.

17. Cuando profundizo en el estudio, luego sé que puedo aplicar en la práctica lo que voy aprendiendo

18. Si puedo, intentaré sacar mejores notas que la mayoría de mis compañeros/as

19. Lo que quiero es estudiar solamente lo que me van a preguntar en los exámenes

20. Prefiero estudiar los temas que me resultan interesantes, aunque sean difíciles

21. Cuando salen las notas acostumbro a compararlas con las de mis compañeros/as o las de mis amigos/as

22. Creo que soy un buen/a estudiante

23. Tengo buenas cualidades para estudiar

24. Me considero un estudiante del montón

\section{Anexo 2: preguntas en el test de motivación Semap-01}

A continuación los Ítems propuestos en la Subescala de SUBESCALA SEAT-01 (Estilos atribucionales) de la Escala CEAP48 propuesta por Barca et al. (2005):

1. Me esfuerzo en mis estudios porque mis padres se sienten orgullosos de mí

2. Siempre que estudio lo suficiente, obtengo buenas notas

3. Cuando el profesorado se preocupa y da directrices de cómo estudiar, entonces me encuentro bien en clase y en los exámenes.

4. Las buenas notas se deben siempre a mi capacidad

5. Mi éxito en los exámenes se debe en gran parte a la suerte

6. Las materias de estudio, en general, son fáciles, por eso obtengo buenas notas

7. Me esfuerzo en mis estudios porque deseo aumentar mis conocimientos y mi competencia profesional futura

8. Normalmente me esfuerzo en mis estudios porque quiero ser valorado por mis amigos y compañeros de clase

9. Estudio desde el principio y lo hago todos los días, así nunca tengo problemas para tener buenas notas

10. El profesorado es el responsable de mi bajo rendimiento académico

11. Cuando fracaso en los exámenes se debe a mi baja capacidad

12. Si obtengo malas notas es porque tengo mala suerte

13. Es fácil para mí comprender los contenidos de las materias que tengo que estudiar para obtener buenas notas

14. Me esfuerzo en los estudios porque me gusta lo que estoy trabajando en clases

15. Me esfuerzo en mis estudios porque quiero obtener las mejores notas de clase

16. Estudio para obtener buenas notas porque es la mejor manera de sobresalir en clase

17. Cuando el profesorado explica bien, me ayuda a obtener buenas notas

18. Cuando obtengo malas notas pienso que no estoy capacitado/a para triunfar en esas materias

19. A veces mis notas me hacen pensar que tengo mala suerte en la vida y especialmente en los exámenes

20. Mis buenas notas reflejan que algunas de las materias que tengo son fáciles

21. Me esfuerzo en mis estudios porque me resulta muy útil ver como lo que sé me sirve para aprender cosas 
nuevas

22. Cuando tengo malas notas es porque no he estudiado lo suficiente

23. Mi fracaso en los exámenes se debe en gran parte a la mala suerte

24. Mis malas notas reflejan que las materias son difíciles. 